Rev. salud pública. 10 (3):423-432, 2008

\title{
Prevalencia y Caracterización de los Recién Nacidos con Anomalías Craneofaciales en el Instituto Materno Infantil de Bogotá
}

\author{
Prevalence and characterisation of the newborn suffering \\ craniofacial anomalies at the Instituto Materno Infantil in Bogotá
}

Yolanda Cifuentes-Cifuentes ${ }^{1}$, Clara Arteaga-Díaz ${ }^{2}$, Clementina InfanteContreras $^{3}$, Elsa G. Clavijo-López ${ }^{3}$ y Constanza Quintero-Guzmán ${ }^{4}$

\begin{abstract}
1 División de Perinatología y Neonatología, Departamento de Pediatría, Instituto Materno Infantil, Facultad de Medicina, Universidad Nacional de Colombia. mycifuentesd@unal.edu.co 2 Departamento de Ginecología y Obstetricia, Facultad de Medicina, Instituto Materno Infantil. Universidad Nacional. cearteagad@unal.edu.co

3 Facultad de Odontología, Universidad Nacional de Colombia. ccontrerasi@unal.edu.co, egclavijol@unal.edu.co.

4 Facultad de Ciencias, Universidad Nacional de Colombia. cquinterog@unal.edu.co
\end{abstract}

Recibido 18 Agosto 2007/Enviado para Modificación 3 Abril 2008/Aceptado 6 Junio 2008

\section{RESUMEN}

Objetivos Determinar la frecuencia y caracterizar los neonatos nacidos con anomalías craneofaciales atendidos en el Instituto Materno Infantil (IMI).

Métodos Se recolectaron los datos de nacidos o remitidos al IMI, estrato, procedencia, factores de riesgo general y específico, género, edad gestacional, correlación peso y edad gestacional, diagnóstico de la anomalía, cariotipo y condición al egreso de los neonatos con anomalías craneofaciales atendidos entre 1 de Marzo de 2000 y 15 de Agosto de 2001.

Resultados La prevalencia de anomalía congénita fue 2,7 \% y de anomalía craneofacial 0,6 \%. De 52 pacientes, 69 \% tenía factores de riesgo específico, $38 \%$ era prematuro y $33 \%$ tenía retardo del crecimiento intrauterino; 65 \% correspondió a hendiduras, 55,8 \% a anomalía múltiple y $46 \%$ a síndromes. Se estableció diagnóstico etiológico en $38 \%$ de los casos; $12 \%$ tenía cromosomopatía. Fallecieron 18 niños (35\%) y en 13 la muerte se atribuyó a la anomalía, 6 de éstos tenían cromosomopatía. Se evidenció la utilidad del cariotipo para hacer diagnóstico etiológico y establecer pronóstico. Los neonatos con anomalías craneofaciales tuvieron 3 veces más riesgo de morir que el resto de neonatos hospitalizados. Se demostró asociación estadísticamente significativa de la mortalidad con prematurez, retardo de crecimiento, cromosomopatía y diagnósticos de anomalía múltiple y síndrome.

Conclusiones Prematurez, retardo de crecimiento, anomalía múltiple, diagnóstico de síndrome y cromosomopatía evidencian un perfil de riesgo para mortalidad. 
Palabras Clave: Anomalías craneofaciales, recién nacido, retardo del crecimiento fetal, prematuro, mortalidad (fuente: DeCS, BIREME).

\section{ABSTRACT}

Objectives Determining the frequency and characteristics of newborn suffering craniofacial abnormalities who were attending the Instituto Materno Infantil (IMI) in Bogotá.

Methods Data was gathered regarding the newborn suffering such alterations who attended IMI from March $1^{\text {st }} 2000$ to August $15^{\text {th }} 2001$. The variables analysed were: being born in or having been remitted to the IMI, social strata, origin, general and specific risk factors, gender, correlation between weight and gestational age, clinical and aetiological diagnosis of the anomaly, karyotype and condition on leaving IMI.

Results There was 2,7\% prevalence for congenital defects and 0,6 \% for craniofacial abnormalities. $69 \%$ of the 52 patients had specific risk factors, $38 \%$ had been preterm, $33 \%$ had suffered retarded intra-uterine growth, $65 \%$ had cleft palates, $55,8 \%$ multiple abnormalities and $46 \%$ syndromes. Aetiological diagnosis was established in $38 \% ; 12 \%$ had chromosomopathy. Eighteen children (35\%) died, death being attributed to their defect in 13 of them, six by chromosomopathy. This study revealed the use of karyotype in making an aetiological diagnosis and determining prognosis. Mortality for the group suffering craniofacial abnormalities was threefold that of other hospitalised neonatal patients. Significant statistical association was shown between mortality and preterm condition, retarded growth, chromosomopathy and a diagnosis of multiple defects and/or syndromes.

Conclusions Pre-maturity, retarded growth, multiple defects, syndrome diagnosis and chromosomopathy revealed a mortality risk profile.

Key Words: Craniofacial abnormality, newborn, retarded foetal growth, premature, mortality (source: $\mathrm{MeSH}, \mathrm{NLM}$ ).

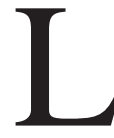

as anomalías congénitas constituyen la segunda causa de mortalidad infantil en nuestro país (1). En Estados Unidos en la década del 90 el 22

\% de la mortalidad infantil ocurrió por esta causa, la tercera parte debida a anomalías incompatibles con la vida: anencefalias, encefaloceles, hipoplasia pulmonar, agenesia renal y trisomías 13 y 18 (2).

Las anomalías craneofaciales representan entre 10 y $15 \%$ de las anomalías congénitas (3-5) siendo las hendiduras orofaciales una de las más comunes y más graves. Su frecuencia es muy variable dependiendo de las poblaciones. Un caso de hendidura orofacial ocurre por cada 500 a 550 nacimientos aproximadamente $(6,7)$. 
En Colombia el estudio de Muñoz muestra una frecuencia de 1:1 015 nacimientos (8). Se consideran dentro de las anomalías craneofaciales las anencefalias, encefaloceles y otros defectos del sistema nervioso central como la holoprosencefalia, con una prevalencia de 1 a 5 por 1000 recién nacidos $(5,9)$. Los defectos de primero y segundo arco branquial, incluidas las microtias con una prevalencia de 0,3-5,5 por $10000(10,11)$ y las craneosinostosis con una prevalencia de 0,6 por $1000 \quad(3,4)$ también hacen parte de las anomalías craneofaciales.

Algunos estudios han sugerido que entre $44 \%$ y $64 \%$ de los pacientes con hendiduras tienen anomalías asociadas, estos cuadros pueden considerarse como síndromes, secuencias, defectos de campo o asociaciones (6). Un estudio realizado en Francia en 238942 nacimientos entre 1979 y 1996 mostró que de 460 recién nacidos con hendiduras, 36,7 \% tenía anomalías asociadas, más frecuentes en los niños con sólo paladar hendido (46,7 \%) que en los niños con labio y paladar hendido (36,8 \%) y que en los niños con labio hendido solamente (13,6 \%) (12).

El encefalocele y la anencefalia, junto con otros defectos de cierre del tubo neural, ocurren en forma aislada en más del $95 \%$ de los casos pero pueden formar parte de un cuadro de múltiples anomalías de origen monogénico, como el síndrome de Meckel Gruber, las trisomías 13 o 18 o por efecto de teratógenos como aminopterina o ácido valproico o fenotipos definidos de causa desconocida como el asociado con extrofia de cloaca $(13,14)$.

La microtia, anotia o fístulas y apéndices preauriculares suelen aparecer en forma aislada entre $45 \%$ y $50 \%$ de los casos y pueden formar parte de cuadros de múltiples anomalías como la asociación Charge, el síndrome de TownesBrocks, el síndrome BOR, el síndrome de Treacher Collins, la embriopatía diabética y el síndrome facio aurículo vertebral. $(15,16)$.

La etiología de las anomalías craneofaciales es heterogénea, pudiendo ser: 1) Monogénica: cerca del $5 \%$ de los síndromes monogénicos reportados en el catálogo de McKusick tienen dismorfia craneofacial severa (17).

2) Cromosómica: la mayoría de las cromosomopatías presenta anomalías craneofaciales, desde moderadas hasta graves; la trisomía 21 o síndrome de Down, cursa con anomalías faciales menores y sólo en el $1 \%$ de los casos con una hendidura labial o palatina (18); en la trisomía 18 la anomalía facial más frecuente es la micrognatia acompañada sólo en el $10 \%$ de los casos de hendiduras labiales o palatinas; en la trisomía 13 el 90 \% de los casos presenta una grave 
dismorfia facial. Aparte de estos grandes síndromes, existen alrededor de 75 patologías debidas a anomalías de cromosomas autosómicos, en todas, la dismorfia facial es la más notable alteración por su especificidad y por su facilidad para reconocerla (19).

3) Multifactorial: la mayoría de las anomalías congénitas de etiología conocida (40-50 \%) tienen causa multifactorial. En este grupo las más frecuentes son las cardiopatías congénitas, seguidas por las hendiduras labiopalatinas y los defectos de cierre del tubo neural.

4) Teratogénica: alrededor del $3 \%$ de las anomalías congénitas se relacionan con teratógenos específicos. De casi 600 agentes teratogénicos conocidos en animales, sólo en 25 \% se ha hallado relación con anomalías en humanos, la mayoría ocasionando una alteración craneofacial reconocible (19).

Este estudio además de los aspectos antes referidos, muestra las características clínicas de los neonatos con anomalías craneofaciales, aspecto fundamental en el manejo de estos pacientes.

\section{MATERIALES Y MÉTODOS}

Se recolectaron los datos de 52 casos atendidos entre el 1 de Marzo de 2000 y el 15 de Agosto de 2001. Las variables lugar de nacimiento, procedencia, estrato, factores de riesgo obstétrico general y específico, sexo del neonato, edad gestacional, correlación de peso para la edad gestacional, diagnósticos clínico, de la anomalía y etiológico, cariotipo, desenlace y causa de muerte fueron recolectadas en un formulario. Se diligenció consentimiento informado con los padres. Se realizó cariotipo en 45 pacientes.

Se consideró riesgo obstétrico general la presencia uno o más de: ausencia de control prenatal, primiparidad, multiparidad (más de tres gestaciones) y patología gestacional diferente a diabetes. Se consideró riesgo obstétrico específico la presencia de uno o más de: edad materna menor o igual a 18 años o mayor o igual a 35 años, edad paterna mayor o igual a 50 años, antecedente de aborto o mortinato, consanguinidad, antecedente familiar de anomalía congénita y/o de anomalía craneofacial, exposición a mutágenos y presencia de diabetes gestacional. Para determinar la presencia de exposición a mutágenos se utilizaron las categorías cdx de la FDA. 
Se consideró neonato pretérmino al que tuviera menos de 37 semanas de edad gestacional y a término entre las 37 y las 42 semanas de acuerdo con la escala de Capurro. Para la correlación de peso con la edad gestacional se utilizó la clasificación de Lubchenco-Bataglia.

Se definió anomalía congénita como cualquier alteración en la estructura corporal presente al nacimiento, anomalía aislada aquella que se presenta como alteración única o acompañada de otra no relacionada, anomalía múltiple cuando hay dos o más anomalías relacionadas entre sí. Cuando la presentación de la anomalía fue múltiple se clasificó como síndrome, secuencia, asociación y defecto de campo de desarrollo. La clasificación de la anomalía se basó en el hallazgo clínico predominante, hendidura, microtia, encefalocele y otros.

Se clasificaron en monogénicas, cromosómicas, multifactoriales y teratogénicas según etiología; si no fue posible establecerla, se consideraron sin clasificar. Se usó la base de datos de London Dysmorphology (17).

Se consideró como desenlace la condición vivo o muerto al egreso del hospital. La muerte se registró como causada por la anomalía, cuando al revisar la historia clínica no se encontró otra patología que la explicara.

Para el análisis se diseñó una base de datos en Excel y se utilizó el programa estadístico SAS. Se probaron las asociaciones entre variables con la prueba Chi cuadrado para independencia de variables categóricas.

\section{RESULTADOS}

Durante este tiempo el IMI atendió 4152 partos y 2916 RN hospitalizados, 1 453 nacidos en el IMI y 1463 remitidos; el 25,5 \% era prematuro, 14,9 \% tenía RCIU y 11,1 \% tenía Apgar inferior a 7. La mortalidad en los neonatos hospitalizados, nacidos en la Institución y remitidos fue de 11,3 \%.

La frecuencia de anomalía craneofacial en los nacidos en la Institución fue de $0,65 \%$, que corresponde a 1 caso por cada 154 nacidos vivos. De los hospitalizados, 52 casos (1,78 \%) tenían anomalía craneofacial, para una frecuencia de 1 por 56 ingresos.

De los 52 casos de anomalía craneofacial, 52 \% nació en el IMI (27 casos), 73 \% (38 casos) era de Bogotá, 79 \% (41 casos) de estrato 2, 31 \% (16 casos) tenía riesgo por edad materna, ninguno por edad paterna, 79 \% asistió a control 
prenatal (41 casos), 65 \% (34 casos) tenía riesgo por paridad, 19 \% (10 casos) tenía antecedentes de aborto o mortinato, 52 \% tenía patología gestacional, 29 \% tenía exposición a mutágenos en el primer trimestre.

El 88,4 \% tenía factores de riesgo obstétrico general, 69 \% tenía factores de riesgo específico, 35 \% (18 casos) tenía antecedente familiar de anomalía congénita y de estos, 14 tenía antecedente familiar de anomalía craneofacial.

De los 52 casos, 44,2 \% presentó anomalía aislada y 55,8 \% anomalía múltiple (Tabla 1).

El mayor número de casos (34) correspondió a hendiduras orales, se encontraron 7 microtias (13,5\%), 5 encefaloceles (9,6 \%) y 6 casos de fístulas preauriculares, criptoftalmia, teratoma, micrognatia, nariz bífida ehipertelorismo. La Tabla 2 muestra las características y diagnósticos de los 52 casos de anomalía craneofacial.

\begin{tabular}{|c|c|c|}
\hline Diagnostico Clinico & $\mathrm{N}$ & $\%$ \\
\hline Aislada & 23 & 44,2 \\
\hline Múltiple & 29 & 55,8 \\
\hline Sindrome & \multicolumn{2}{|c|}{24} \\
\hline Secuencia & \multicolumn{2}{|c|}{3} \\
\hline Defecto de campo & \multicolumn{2}{|c|}{1} \\
\hline As $\propto c i a c i o ́ n$ & \multicolumn{2}{|c|}{1} \\
\hline Total & 52 & 100 \\
\hline
\end{tabular}

En 20 casos (38 \%) no se pudo establecer el diagnóstico etiológico, 14 casos (27\%) fueron de origen multifactorial, 11 (21 \%) monogénico, 6 (12 \%) cromosómico y 1 teratogénico.

De los 17 casos de RCIU, 13 casos (76 \%) tuvieron anomalías múltiples y solamente 4 casos (24\%) presentaron anomalía aislada.

De 29 pacientes con anomalía múltiple, 21 casos (72 \%) tuvieron cariotipo normal, 6 casos (21 \%) tuvieron cromosomopatía y a 2 casos $(7 \%)$ no se les realizó cariotipo. De 23 pacientes con anomalía aislada, 18 casos (78 \%) tuvieron cariotipo normal, ningún caso tuvo cromosomopatía y a 5 casos (22 \%) no se les realizó cariotipo.

Fallecieron 18 casos (35 \%), 13 a causa de la anomalía congénita. La Tabla 3 muestra las características de los casos de mortalidad. 
Tabla 2. Distribución de los 52 pacientes según el diagnóstico de la anomalía

\begin{tabular}{|c|c|c|c|c|}
\hline & Hendiduras & Microtias & $\begin{array}{l}\text { Encefalo } \\
\text { celes }\end{array}$ & Otros \\
\hline Casos & 34 & 7 & 5 & 6 \\
\hline Femenino & 19 & 1 & 4 & 1 \\
\hline Masculino & 15 & 6 & 1 & 5 \\
\hline Pretérmino & 15 & 1 & 2 & 2 \\
\hline Retardo de crecimiento & 9 & 2 & 3 & 3 \\
\hline Factores riesgo general & 29 & 6 & 5 & 6 \\
\hline Factores riesgo específico & 20 & 5 & 5 & 6 \\
\hline $\begin{array}{l}\text { Antecedente.Familiar anomalía } \\
\text { congenital }\end{array}$ & 10 & 4 & 1 & 3 \\
\hline $\begin{array}{l}\text { Antec edente Familiar anomalía } \\
\text { craneofacial }\end{array}$ & 8 & 3 & 1 & 2 \\
\hline Anomalía aislada & 15 & 4 & 3 & 1 \\
\hline Anomalía múltiple & 19 & 3 & 2 & 5 \\
\hline Sindrome & 16 & 3 & 1 & 4 \\
\hline Secuencia & 1 & & 1 & 1 \\
\hline Defec to de campo & 1 & & & \\
\hline As ociación & 1 & & & \\
\hline Con diagnóstico etiológico & 27 & 3 & 0 & 2 \\
\hline Monogénico & 7 & 2 & & 2 \\
\hline Anomalía cromosómica & 5 & 1 & & \\
\hline Multifactorial & 14 & & & \\
\hline Teratogénico & 1 & & & \\
\hline Sin diagnóstic o etiológico & 7 & 4 & 5 & 4 \\
\hline Muertes & 16 & 1 & 0 & 1 \\
\hline Muertes por la anomalía & 11 & 1 & 0 & 1 \\
\hline
\end{tabular}

Tabla 3. Descripción de los 18 casos de mortalidad (\%)

\begin{tabular}{|c|c|c|c|}
\hline Casos & & $\mathrm{N}$ & $\%$ \\
\hline Femenino & & 11 & 61,1 \\
\hline Pretérmino & & 11 & 61,1 \\
\hline Retardo de crecimiento & & 9 & 50 \\
\hline Nacidos en el IMI & & 12 & 66,6 \\
\hline Remitidos & & 6 & 33,3 \\
\hline Factores riesgo general & & 17 & 94,4 \\
\hline Factores riesgo espec ífico & & 12 & 66,6 \\
\hline $\begin{array}{l}\text { Antecedente familiar anomalía } \\
\text { congénita }\end{array}$ & & 5 & 27,7 \\
\hline $\begin{array}{l}\text { Antecedente familiar anomalía } \\
\text { craniofacial }\end{array}$ & & 4 & 22,2 \\
\hline \multirow[t]{3}{*}{ Diagnóstico de la anomalia } & Hendiduras & 16 & 88,8 \\
\hline & Microtias & 1 & 5,5 \\
\hline & Otros (Teratoma) & 1 & 5,5 \\
\hline \multirow[t]{6}{*}{ Diagnóstico clínico } & Aislada & 3 & 16,6 \\
\hline & Múltiple & 15 & 83,3 \\
\hline & Síndromes & 12 & \\
\hline & Defecto de campo & 1 & \\
\hline & Secuencia & 1 & \\
\hline & Asociación & 1 & \\
\hline \multirow[t]{5}{*}{ Diagnóstico etiológico } & Cromosomopatía & 6 & 33,3 \\
\hline & Monogénico & 3 & 16,6 \\
\hline & Multifactorial & 3 & 16,6 \\
\hline & Teratogénico & 1 & 5,5 \\
\hline & Sin clasificar & 5 & 27,7 \\
\hline Muertes por la anomalía & & 13 & 72,2 \\
\hline
\end{tabular}


De los 23 pacientes con anomalía aislada fallecieron 3 (13\%) mientras que de los 29 casos de anomalía múltiple fallecieron 15 (51,7 \%). Esta diferencia se hace más evidente si se considera la causa de mortalidad, pues en el grupo de anomalía aislada no hubo ningún caso de mortalidad por la anomalía, por el contrario en el grupo de anomalía múltiple, 13 de las 15 muertes, se debieron a la anomalía, de éstos 6 correspondían a la totalidad del grupo de cromosomopatía (tres trisomías 13, dos trisomías 18 y una trisomía 21).

La distribución de las variables sexo, edad gestacional, peso para la edad gestacional, cromosomopatía, factores de riesgo general, factores de riesgo específico, antecedente familiar de anomalía congénita y craneofacial, desenlace y causa de muerte es similar cuando se comparan los 32 casos con diagnóstico etiológico y los 20 sin diagnóstico etiológico y los 23 casos con anomalía aislada con los 29 casos con anomalía múltiple.

\section{DISCUSIÓN}

Tal como está informado en la literatura el mayor número de pacientes correspondió a hendiduras orofaciales. El 55,8 \% de los casos correspondió a anomalías múltiples, mayor que el reportado en la literatura lo cual puede ser explicado por ser un centro de remisión.

Un caso de encefalocele presentó múltiples anomalías, diagnosticadas como efecto disruptivo de bridas amnióticas, los demás fueron aislados, dos casos de microtia hacían parte del síndrome de Goldenhar y un caso sindrómico de hendidura correspondió a un síndrome de pterigio poplíteo.

Llama la atención la frecuencia de anomalía cromosómica (12%) al compararla con la informada por la literatura, así como la frecuencia de la anomalía multifactorial, que en nuestro estudio fue del $27 \%$ y la informada es del $50 \%$. Solo en un caso se encontró un teratógeno que podría ocasionar la anomalía.

La literatura consultada no informa sobre antecedente familiar de anomalía congénita. En nuestro estudio 18 de 52 (35 \%) de los pacientes tenían el antecedente y 14 de éstos tenían antecedente de anomalía craneofacial; Dado que la etiología de la anomalía craneofacial puede incluir factores genéticos familiares es necesario que este aspecto se incluya dentro del interrogatorio. 
El 38,4 \% de los pacientes era pretérmino y 32,7 \% tenía RCIU, en ese período la frecuencia de prematurez en el IMI fue 25,5 \% y de RCIU 14,9 \%, estas diferencias son estadísticamente significativas $(\mathrm{p}<0.05)$.

La mortalidad en los niños hospitalizados por patología neonatal fue $11,3 \%$, en los recién nacidos con anomalía craneofacial la mortalidad fue 34,6 \% siendo esta diferencia estadísticamente significativa, mostrando que los niños que se hospitalizan con anomalía craneofacial tienen 3 veces más de riesgo de morir comparada con los niños que se hospitalizan por patología neonatal de común ocurrencia.

En el grupo de pacientes a quienes se les realizó cariotipo, ningún paciente con anomalía aislada tuvo cromosomopatía pero más de la quinta parte de los pacientes con anomalía múltiple, presentó cromosomopatía. Es evidente que existe una diferencia entre estos dos grupos respecto a la cromosomopatía, corroborando que, el cuadro clínico de las alteraciones cromosómicas se presenta casi siempre con múltiples anomalías.

De 13 pacientes fallecidos con diagnóstico, 6 correspondían a la totalidad del grupo de cromosomopatía, que evidencia la necesidad de realizar cariotipo y la gravedad cuando el diagnóstico es anomalía cromosómica.

De 35 casos que no presentaron RCIU, 19 (54\%) tuvieron anomalías aisladas y 16 casos (46 \%) múltiples, de los 17 casos de RCIU, 13 casos (76 \%) tuvieron anomalías múltiples y solamente 4 (24\%) presentaron anomalía aislada, esta diferencia es estadísticamente significativa, $X^{2}=4,38$ y $\mathrm{p}<0,05$. De los 23 pacientes con anomalía aislada fallecieron $3(13 \%)$ y de los 29 casos de anomalía múltiple 15 (51,7\%), esta diferencia es estadísticamente significativa con $\mathrm{p}<$ 0,005 y un valor de $X^{2}$ de 8,4794 . La diferencia de mortalidad se hace más evidente si se considera causa de mortalidad, pues en el grupo de anomalías aisladas no hay ningún caso de mortalidad por la anomalía, por el contrario en el grupo de anomalía múltiple, 13 de las 15 muertes, se debieron a la anomalía. Es fundamental definir si se trata de una anomalía aislada o de un cuadro de múltiples anomalías, para determinar gravedad, pronóstico y evolución de un paciente con anomalía craneofacial.

La prematurez, el retardo de crecimiento, la anomalía múltiple, el diagnóstico de síndrome, la cromosomopatía, evidencian un perfil de riesgo para mortalidad, que para el manejo clínico es útil y de fácil reconocimiento 
Agradecimientos. La publicación se hace como un homenaje al Instituto Materno Infantil.

\section{REFERENCIAS}

1. Biblioteca Virtual para la Vigilancia en Salud Pública de Colombia. Indicadores básicos de salud [Internet]. Disponible en http://www.bvsvspcol.bvsalud.org/xml $2 \mathrm{html} /$ xmlRoot.php?xml=xml/es/bvs. $\quad x m l \& x s l=x s l /$ level2.xsl\&lang=es\&tab=collection\&item=132\&graphic=yes Consultado en junio de 2007.

2. Lee K, Khoshnood B, Chen L, Wall SN, Cromie WJ. Mittendorf RL. Infant Mortality from congenital malformation in the United States 1970-1997. Obstet Gynecol 2001; 98(4):620-7.

3. Hunter AG, Rudd NL. Craniosynostosis I. Sagittal synostosis: Its genetics and associated clinical findings in 214 patients who lacked involvement of the coronal sutures. Teratology 1976; 14(2):185-193.

4. Shuper A, Merlob P, Grunebaum M, Reisner SH. The incidence of isolated craniosynostosis in the newborn infant. Am J Dis Child. 1985; 139(1):85-6.

5. Lemire RJ. Anencephaly. In: Vinken PJ, Bruyn GW (eds). Handbook of Clinical Neurology: Malformations, New York : Elsevier; 1987.

6. Cervenka J, Tolarova M. Classification and Birth Prevalence of Orofacial Clefts. Am. J. Med. Genet. 1998; 75:126-137.

7. Ranta R, Tulensalo T. Symmetry and combinations of hipodontia in non-clef and cleft palate childrens. Scand. J. Dent. Res. 1988;96:1-8.

8. Muñoz J, Bustos I, Quintero C, Giraldo A. Factores de riesgo para algunas anomalías congénitas en población colombiana. Rev Sal. Pub. 2001; 3(3):268-82.

9. Rankin J, Glinianaia S, Brown R, Renwick M. The changing prevalence of neural tube defects: A population based study in the north of England, 1984-96. Paediatr Perinat Epidemiol 2000; 14(2):104-110.

10. Poswillo D. Otomandibular deformity: pathogenesis as a guide to reconstruction. J Maxilofac surg. 1974; 2:64-72.

11. Poswillo D. Hemorrhage in development of the face. Births defects Orig Artic Ser 1975; 11(7):6181.

12. Stoll C, Alembik Y, Dott B, Roth MP. Associated malformations in cases with oral clefts. Cleft Pal Cran Jour 2000; 37(1):41-47.

13. Holmes LB, Driscoll SG and Atkins L. Etiologic heterogeneity of neural-tube defects. N Engl J Med 1976; 294:365-369.

14. Volpe JJ. Neurology of the newborn Third Edition. Philadelphia: W.B. Saunders Co; 1995.

15. Wang RY, Earl DL, Ruder RO, Graham JM Jr. Syndromic ear anomalies and renal ultrasounds. Pediatrics 2001; 108(2):E32.

16. Llano-Rivas I, González del Angel A, del Castillo V, Reyes R, Carnevale A. Microtia: A clinical and genetic study at the National Institute of Pediatrics in Mexico City. Arch Med Res 1999; 30(2):120-124.

17. London dysmorphology. Data 3. Oxford University Press; 2002.

18. Kallen B, Mastroiacovo P, Robert E. Malformations in Down Syndrome Am J Med Genet 1996; 65(2):160-166.

19. Guizar JJ. Genética Clínica. Diagnóstico y manejo de las enfermedades hereditarias. Tercera Edición. México, Manual Moderno; 2001. 